\title{
Channel and Error Modeling for Wireless Body Area Networks
}

\author{
Saeed Rashwand \\ Department of Computer Science \\ University of Manitoba
}

\author{
Jelena Mišić \\ Department of Computer Science \\ Ryerson University
}

\begin{abstract}
Wireless Body Area Networks (WBANs) have been developed as the human-body monitoring systems to predict, diagnose, and treat diseases. Since the signal transmission in WBANs takes place in or around the human body the channel fading significantly affects packet error rate and overal network performance. In this work, we discuss the channel models and error performance formalization for WBANs. In the first phase of this work, we study channel fading models for WBANs. In the second phase, we survey the models which calculate the error performance metrics in WBANs. We select most appropriate error models to design and develop the error performance evaluation models for IEEE 802.15.6-based WBANs and show how to integrate them with the error model in Medium Access Control (MAC). We then discuss integrated PHY and MAC error performance in WBANs.
\end{abstract}

\section{INTRODUCTION}

A WBAN is a novel wireless technology-driven human body monitoring network which aims to predict and diagnose any diseases and monitor the response of the body to treatments. The network is composed of small and intelligent wireless medical sensors which are worn or implanted into the tissues. The collected data is transmitted to a medical center to be further processed and stored. WBANs must support the combination of reliability, Quality of Service $(\mathrm{Q} Q \mathrm{~S})$, low power, high data rate and non-interference to address the breadth of WBAN applications. Hence, the IEEE 802.15 Working Group developed the IEEE 802.15.6 standard which is optimized for low power devices and operation on, in or around the human body [1]. The IEEE 802.15.6 standard was approved in 2012 for wireless communications in WBANs.

Since the signal transmission in WBANs takes place around or in the human body, the channel fading significantly affects the network error performance. The evaluation of WBAN error performance can be divided into two parts. The WBAN channel characterizations and modeling is the first part of the WBAN error performance investigation. The radio wave propagation in a WBAN is noticeably different compared to the other environments since it takes place inside or close to the human body. WBAN channel modeling is challenging due to the complex shape and composition of the human body. In addition, according to the recent studies, the propagating wave is mostly diffracted around the human body rather than passing 
through it. Hence, the path loss in WBANs is very high specially when the transmitter and the receiver are shadowed by a part of the body [2]. The channel modeling is an important phase of the WBAN error performance investigation, which will be reviewed in Section III. Analysing the error performance of the WBAN communication system for the combination of communication type (modulation/detection), the channel fading and/or co-channel interference is the second major research phase. In this phase of the WBAN error performance investigation the Bit Error Rate (BER) and Packet Error Rate (PER) for all WBAN User Priorities (UPs) are formalized and calculated. In this work, we review the BER or PER modelling of wireless communication systems which could be considered for error performance investigation of IEEE 802.15.6-based WBANs. The second part of the WBAN error performance evaluation will be surveyed in Section IV.

The remainder of this paper is organized as follows: In Section II we describe the wireless signal transmissions in WBANs. Section III discusses the channel fading models for WBANs. In Section IV, we study the error models for calculating BER and error performance of WBANs. In Section V we discuss the cross layer MAC error performance modelling for WBANs. In this section, we discuss integration of PHY and MAC layer in IEEE 802.15.6-based WBAN. Finally, Section VI concludes the paper.

\section{WIRELESS SIGNAL TRANSMISSION IN WBANS}

IEEE 802.15.6 [1] defines a standard for WBANs which is a short range, low power, and highly reliable wireless communication in the vicinity of, or inside, a human body. The standard is designed to support the advanced medical and entertainment options. It is developed so that medical equipment and consumer electronics manufacturers have small, power efficient, inexpensive solutions for implementation of a wide range of devices.

A. IEEE 802.15.6-based WBAN channels

TABLE I

FREQUENCY BAND DEPENDENT PARAMETERS

\begin{tabular}{|c|c|c|c|c|c|c|c|}
\hline Frequency Band (MHz) & $\begin{array}{l}402 \\
405\end{array}$ & $\begin{array}{l}420 \\
450\end{array}$ & $\begin{array}{l}863 \\
870\end{array}$ & $\begin{array}{l}902 \\
928\end{array}$ & $\begin{array}{l}950 \\
956\end{array}$ & $\begin{array}{l}2360 \\
2400\end{array}$ & $\begin{array}{l}2400 \\
2483.5\end{array}$ \\
\hline Symbol Rate (ksps) & 187.5 & 187.5 & 250 & 300 & 250 & 600 & 600 \\
\hline number of channels & 10 & 12 & 14 & 48 & 12 & 38 & 79 \\
\hline Channel Bandwidth (MHz) & 0.30 & 0.50 & 0.40 & 0.50 & 0.40 & 1.00 & 1.00 \\
\hline
\end{tabular}


Based on the IEEE 802.15.6 standard, the WBANs can operate in an extensive set of wireless channels as given in Table I. The channel-related parameters are also shown in the Table I. The selected channel in which the WBAN operates considerably affects the network performance. The channel fading and the WBAN error performance varies by changing the operating channel.

TABLE II

DATA RATE DEPENDENT PARAMETERS

\begin{tabular}{|c|c|c|c|c|c|}
\hline $\begin{array}{l}\text { Frequency Band } \\
(\mathrm{MHz})\end{array}$ & Packet Component & Modulation & $\begin{array}{l}\text { Spreading } \\
\text { Factor(S) }\end{array}$ & $\begin{array}{l}\text { Information } \\
\text { Data Rate } \\
(\mathrm{kbps})\end{array}$ & Support \\
\hline $402-405$ & PLCP Header & $\pi / 2$-DBPSK & 2 & 57.5 & Mandatory \\
\hline $402-405$ & PSDU & $\pi / 2$-DBPSK & 2 & 75.9 & Mandatory \\
\hline $402-405$ & PSDU & $\pi / 2$-DBPSK & 1 & 151.8 & Mandatory \\
\hline $402-405$ & PSDU & $\pi / 4-\mathrm{DQPSK}$ & 1 & 303.6 & Mandatory \\
\hline $402-405$ & PSDU & $\pi / 8$-D8PSK & 1 & 455.4 & Optional \\
\hline 420-450 & PLCP Header & GMSK & 2 & $\overline{57.5}$ & Mandatory \\
\hline $420-450$ & PSDU & GMSK & 2 & 75.9 & Mandatory \\
\hline $420-450$ & PSDU & GMSK & 1 & 151.8 & Mandatory \\
\hline $420-450$ & PSDU & GMSK & 1 & 187.5 & Optional \\
\hline $863-870$ & PLCP Header & $\pi / 2$-DBPSK & 2 & 76.6 & Mandatory \\
\hline $863-870$ & PSDU & $\pi / 2$-DBPSK & 2 & 101.2 & Mandatory \\
\hline $863-870$ & PSDU & $\pi / 2$-DBPSK & 1 & 202.4 & Mandatory \\
\hline $863-870$ & PSDU & $\pi / 4-\mathrm{DQPSK}$ & 1 & 404.8 & Mandatory \\
\hline $863-870$ & PSDU & $\pi / 8$-D8PSK & 1 & 607.1 & Optional \\
\hline 90 & PLCP Header & $\overline{\pi / 2 \text {-DBPSK }}$ & 2 & 91.9 & Mandatory \\
\hline $902-928$ & PSDU & $\pi / 2$-DBPSK & 2 & 121.4 & Mandatory \\
\hline $902-928$ & PSDU & $\pi / 2$-DBPSK & 1 & 242.9 & Mandatory \\
\hline $902-928$ & PSDU & $\pi / 4-\mathrm{DQPSK}$ & 1 & 485.7 & Mandatory \\
\hline $902-928$ & PSDU & $\pi / 8$-D8PSK & 1 & 728.6 & Optional \\
\hline $950-956$ & PLCP Header & $\pi / 2$-DBPSK & 2 & 76.6 & Mandatory \\
\hline $950-956$ & PSDU & $\pi / 2$-DBPSK & 2 & 101.2 & Mandatory \\
\hline $950-956$ & PSDU & $\pi / 2$-DBPSK & 1 & 202.4 & Mandatory \\
\hline $950-956$ & PSDU & $\pi / 4-\mathrm{DQPSK}$ & 1 & 404.8 & Mandatory \\
\hline $950-956$ & PSDU & $\pi / 8$-D8PSK & 1 & 607.1 & Optional \\
\hline $2360-2400$ & PLCP Header & 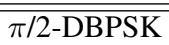 & $\overline{44}$ & 91.9 & Mandatory \\
\hline $2360-2400$ & PSDU & $\pi / 2$-DBPSK & 4 & 121.4 & Mandatory \\
\hline $2360-2400$ & PSDU & $\pi / 2$-DBPSK & 2 & 242.9 & Mandatory \\
\hline $2360-2400$ & PSDU & $\pi / 2$-DBPSK & 1 & 485.7 & Mandatory \\
\hline $2360-2400$ & PSDU & $\pi / 4-\mathrm{DQPSK}$ & 1 & 971.4 & Mandatory \\
\hline $2400-2483.5$ & PLCP Header & $\pi / 2$-DBPSK & $\overline{4}$ & 91.9 & Mandatory \\
\hline $2400-2483.5$ & PSDU & $\pi / 2$-DBPSK & 4 & 121.4 & Mandatory \\
\hline $2400-2483.5$ & PSDU & $\pi / 2$-DBPSK & 2 & 242.9 & Mandatory \\
\hline $2400-2483.5$ & PSDU & $\pi / 2$-DBPSK & 1 & 485.7 & Mandatory \\
\hline $2400-2483.5$ & PSDU & $\pi / 4-\mathrm{DQPSK}$ & 1 & 971.4 & Mandatory \\
\hline
\end{tabular}

The data rate dependent parameters for each of the possible operational frequency bands are provided in Table II. In the table, PLCP, PSDU, DBPSK, DQPSK, and D8PSK, GMSK stand for Physical Layer Convergence Procedure, PHY Service Data Unit, Differential Binary Phase Shift Keying, Differential Quadrature Phase Shift Keying, Differential Eight-Ary Phase-Shift Keying, and Gaussian Minimum Shift 
Keying, respectively [3]. The modulation scheme and the transmission rate affects the error performance of WBANs.

\section{B. Antennas for WBAN transmitter/receiver}

Deploying an appropriate antenna for devices in WBANs is a highly effective parameter on the network error performance. Creeping waves are known as the main propagation approach for signal propagation around the human body due to the very high signal attenuation in the human tissue. Hence, an antenna with a high profile which is polarized perpendicularly to the surface of the body outperforms an antenna with a low profile. Monopoles achieve the highest gain for the devices at the cost of a high profile which is not desirable for WBAN applications. Among all the antennas including monopoles, microstrip patches, Planner Inverted-F Antennas (PIFA), loops, and dipoles, it is believed that the PIFA antennas offer a reasonable compromise between practicality and performance for con-body communications. The PIFA antennas achieve the second best gain performance having higher shadowing losses and efficiency reductions compared to the monopoles [4], [5].

\section{Wireless Interference}

In wireless communications, the radio channel between a transmitter (TX) and a receiver (RX) is the transmission medium. Signal transmission in the wireless communication systems is affected by the two major sources of degradation; multipath fading and Co-Channel Interference (CCI). Multipath fading is caused by travelling the radio frequency $(\mathrm{RF})$ signals between a transmitter and a receiver via a number of different propagation paths. The fading leads the fluctuations in the amplitude, phase, and angle of the received signal. Reflection, diffraction, and scattering are the three major physical phenomena which

affect the radio propagation. In the presence of a strong Line Of Sight (LOS) between the transmitter and the receiver, the diffraction and scattering could be ignored since they do not affect considerably the radio waves. Otherwise, these two radio propagation mechanisms become the dominant factors in the signal transmission. The different MultiPath Components (MPCs) are not distinguishable for a simple receiver since they are just added up which interfere with each other [6], [7]. 


\section{Diversity Techniques}

Since the channel fading in WBANs is quite strong and significantly affects the quality of the received signal, diversity is an important mechanism to improve the error performance of the networks. Diversity techniques, including time, space, frequency, angle, polarization, and antenna (spatial), etc types, are deployed to transmit radio signals over independent fading channels. The diversity employment by the receiver improves the Signal to Noise Ratio (SNR) and thus the average Bit Error Rate (BER). Two major diversity combining techniques include Selection Combining (SC) and Maximum Ratio Combining (MRC). SC achieves much lower diversity gain than MRC, by avoiding to not considering all signals. MRC weighs the signals from different branches according to their SNRs. Another diversity combining is Equal Gain Combining (EGC), in which the phase is corrected while there is no weighting. Considering the benefits of the SC/MRC techniques a new diversity combining technique, called Generalized SC (GSC), was developed. GSC decreases the complexity of MRC technique with the trade-off of some performance degradation [6], [8], [9].

MRC is a commonly used and optimum diversity technique for coherent detection, in which the phase reference with the received signal is required. MRC is well suited to equal energy signals such as Phase Shift Keying (PSK), which is used in WBAN channels. EGC can also be used for the constant envelope signals such as PSK, having the advantage of not requiring knowledge of the channel fading amplitudes. In these cases, EGC technique seems the most appropriate diversity combining technique [10].

\section{E. Channel Fading}

Based on the different spatial scales and the mobility pattern of the transmitter and the receiver there are two main fading effects; small-scale fading and large-scale fading. Over short distances received power slowly fluctuates around the mean. These fluctuations, which are in the order of one wavelength, are called small-scale fading. Another kind of signal variation, the large-scale fading, is often caused by means of shadowing by large and impenetrable objects for the signal. Both small-scale and large-scale fading can be described by a mean and the statistics of the fluctuations around the mean [6]. 


\section{Channel Modeling for WBANs}

In wireless communications, it is well proved that the Rayleigh distribution is the best choice to model the envelope of the received signals when there are a large number of reflective and scattered paths with no LOS signal components. The Rice distribution is used to model the propagation environment where there are dominant non-fading components, such as a LOS propagation path. The Rayleigh distribution is a special case of Rice distribution and occurs when the Rician $\mathrm{K}$ factor is equal to zero. Nakagami-m distribution is known to be the best option for modeling the fading channel conditions that are severer than the Rayleigh distribution. The radio signals in WBANs may experience both types of small-scale and large-scale fading. However, in WBANs the small-scale fading is dominant compared to the large-scale fading due to the short distance between the transmitter and the receiver [7], [10], [11].

Propagation Path Gain (PG) is a common parameter to model the wireless channels. PG is defined as the ratio of the received to the transmitted power of the antennas. For a WBAN, the channel's PG depends on geometry and contents of the human body, the surrounding environment, properties of antennas and their interaction with the body (antenna directivity, mismatch loss, antenna efficiency, launching efficiency of creeping waves). The body movement, the proximity of the antennas to the body, and the dielectric properties of the body tissues near the antenna are effective parameters on the error performance of WBAN communications [4].

There is a small body of research to model the fading channels for WBANs using mathematical, simulation, or experimental models. We present the studies in this area which could be considered for channel characterization and modeling of WBANs. There is not an agreement between the results of different studies since they have arrived to different conclusions.

It is claimed that the Lognormal distribution describes the path-loss fading of WBAN channels well since the human body is the dominating shadowing factor in WBANs [12]. The results of [2] confirm that the Lognormal distribution is the best choice for modeling the small-scale fading in WBANs because there are only a small number of multi-path components from the diffraction around the body.

In [13], a WBAN channel was modelled using temporal sliding window for the simple activity of walking in an office environment where several antennas and positions were considered. The authors' 
results indicate that the small-scale fading and the large-scale fading follows Rician distribution and Lognormal distribution, respectively. The results of [14] validate the findings of [13] showing that the small-scale and large-scale fading of on-body channels are well described by Rician and Lognormal distributions, respectively. The Nakagami-m and Gamma distributions were found to be the second best suited for modeling the smale-scale fading and large-scale fading of WBAN channels.

However, Nakagami-m was shown to be the best option to model the small-scale fading of on-body channels in [12]. The authors in [15], [16] have shown that the small-scale fading follow Rician distribution while Gamma distribution was found the best candidate to model the large-scale fading of WBAN channels considering varieties of activities. There are other studies in the literature indicating that Rician distribution is the best option for WBAN channel characterization and modeling [17], [18].

According to the available studies, we come to the conclusion that Rician distribution is an appropriate option for modeling the small-scale fading of WBAN channels. In addition, either Lognormal or Nakagami$\mathrm{m}$ distributions are the best choices for modeling the large-scale fading.

\section{Physical Layer ERror Modeling For WBANs}

In this section, we discuss the error performance of wireless communication systems. Our focus is on the error performance of wireless body area network channels.

Radio propagation modes, such as LOS radiation, reflections, diffraction, and scattering caused by the objects in the environment (channel fading) and the co-channel interference in the environment make the error modeling performance of the wireless communication systems often a complex task. The physical properties of propagation environments and sophisticated statistical distribution of path loss impose a huge difficulty in the error performance of the wireless systems [7].

To analyse the error performance of a wireless communication system for each combination of communication type (modulation/detection) and the channel fading and/or co-channel interference the average Bit Error Rate (BER) or the average Symbol Error Rate (SER) of the system is calculated. The statistical calculations of either BER or SER at the physical layer are utilized to compute the Packet Error Rate (PER), the data frame error rate, at the higher layers. PER is considered the most important error performance parameter of the wireless systems from viewpoint of the network protocol developers and algorithm 
designers to analyse the end to end performance at the packet level. In this section, we only review the currently available BER or SER modeling of wireless communication systems which are worth considering for WBANs. PER values could be easily computed based on the BER or SER values.

The error performance models of the wireless communication systems are divided into two categories: analytical models and empirical distribution-based models. By means of the analytical models, the average BER for a digital communication system is described by a mathematical expression. It is often a closedform expression which could be solved to calculate the average BER, usually based on the Signal to Noise Ratio (SNR). However, for some cases the expression is in form of a single or multiple integrals with finite limits, which can be numerically integrated to evaluate the system error performance. Since the results of the analytical models are inaccurate due to errors in the approximations and simplifications of the communication channels, the empirical distribution-based models came into existence. In the latter type of models, the error rate data are collected from the real wireless communication experiments for different communication types and physical characteristics. The error is then utilized to develop error probability distributions by numeric integration [7], [10].

\section{A. Analytical models based on finite integration}

In this section, we describe the analytical models for error performance applicable for IEEE 802.15.6based WBANs. According to the standard [1], the only supported modulation schemes for WBANs are BPSK, QPSK, 8PSK, and GMSK and we will describe analytical models for error calculation for these schemes.

The analytical models are divided into two groups; the group which calculates the average BER for general cases and the other group which computes the exact average BER for specific channel model and modulation/detection combination. The former is often in the form of a single or multiple integral with finite limits while the latter is often a closed-form equation for calculating the exact BER for the system. In this section, we first describe the BER performance models of a wireless communication system over fading channels. We then introduce the models which only consider co-channel interference for the wireless communication systems. Finally, we discuss the error performance models for the case where 
both fading channels and co-channel interference exist in the network. As expected, the error performance of the system is a function of the SNR of the system.

There are many studies which develop an analytical model for error performance of a wireless communication system over fading channels without considering the co-channel interference. The authors in [19] developed an expression for computing BER of DQPSK over a slow Rician fading channel. The expression is composed of an infinite series of finite integral terms. The expression was reduced to a closed-form BER equation for DQPSK assuming a Rayleigh fading channel when the ratio of LOS to multipath power is equal to zero. In [20] a new expression was developed to compute BER of DQPSK signals over a Nakagami-m and Additive White Gaussian Noise (AWGN) fading channel. The expression is an approximation involving a single integral with finite limits. In [21] a unified model was introduced for BER of DQPSK in slow fading including Rician, Rayleigh, Nakagami-m and AWGN. The developed equation is an approximation of BER composed of a single integral with finite limits. A closed-form expression of BER of a DBPSK non-coherent receiver with EGC diversity over a Nakagami-m fading channel was developed in [22]. A similar study was performed in [23] which developed a closed-form expression of BER for DBPSK receivers. The difference between [22] and [23] is that the former considers independent and identically distributed fading channels while the latter releases the assumption.

By deploying the alternate representations of classic functions, such as Gaussian and Marcum Qfunctions, the authors in [10], [24] developed a unified framework for evaluating the error performance of coherent, differentially coherent, and noncoherent communications over generalized fading channels. The model is general enough to cover all the typical fading channels. Based on the unified model, an expression is achieved for every combination of communication (modulation/detection) type and channel fading channel. The model can be simplified to a closed-form expression in many cases while in the other cases a single integral with finite limits is obtained for the error probability performance of the communication system. The developed model is general enough not to assume i.i.d fading among the multi-channels where the different channels might be correlated and non-identically distributed (generalized fading channel). In this work, a closed-form formula is obtained for computing the average BER for binary signals (such as BPSK) and a receiver which implements diversity combining. The SER performance expressions in 
case of M-ary signals are calculated using single integrals with finite limits. The BER equations can be obtained based on the above expressions and the modulation schemes.

In [25] an expression is developed for computing the average BER of binary modulated signals for a multichannel coherent receiver. The obtained expression for BER is a single integral with finite limits, either in case of independent or dependent Rician fading channels. The average BER is analysed for MPSK modulation over Rician fading channels considering the linear diversity in [26]. The authors in [27], [28] drive closed-form expressions for calculating the average BER for a class of modulation schemes over Rician fading channels.

The authors in [29], [11] computed the exact BER and SER of linearly modulated signals, including DBPSK, DQPSK, and D8PSK, in Rician, Rayleigh, and Nakagami-m fading channels for coherent receivers with $L$-th order MRC diversity. The considered fading channel is in the form of multiplicative distortion with AWGN. The developed expressions are in the form of single integrals which could be numerically integrated. The results of the work indicate that the error performance is proportional to the inverse of SNR powered by L. We briefly present the obtained expressions for BER and SER based on SNR in [11]. The expressions are developed for the linearly modulated signals with any arbitrary bit mappings, such as Gray or binary coding. We will deploy the expressions in our analytical models of the IEEE 802.15.6 standard in this paper.

Values $s_{t}$ and $\sigma_{t}^{2}$ are defined as the non-zero mean and variance of the $t$-th channel coefficient, which is a complex Gaussian process, per real and imaginary components. The Rice factor, $K_{R}=\left(s^{2} / 2 \sigma^{2}\right)$, is defined as the ratio of the fixed scattered power to the random scattered powers where $\sigma_{t}^{2}=\sigma^{2}$ and $s_{t}=s$ for $t=1 . . L$ since the channels are assumed identically distributed. $\overline{\gamma_{c}}=\frac{1}{2 N_{0}}$ represents the average SNR per channel where $N_{0}$ is the power spectral density of the complex Gaussian random processes for the channels.

The SER of M-PSK in Rician channel is computed as follows:

$$
S E R_{M P S K-\text { Rice }}=G\left(0, \pi-\frac{\pi}{M}, \overline{\gamma_{c}}, L, K_{R}, \sin \frac{\pi}{M}\right)
$$


where

$$
\begin{aligned}
& G\left(\theta_{1}, \theta_{2}, \bar{\gamma}, L, K_{R}, d\right)= \\
& \frac{e^{-L K_{R}}}{\pi} \int_{\theta_{1}}^{\theta_{2}} \frac{\exp \left(\frac{L K_{R}}{1+\left(\bar{\gamma} d^{2} /\left(K_{R}+1\right) \sin ^{2} \theta\right)}\right)}{\left[1+\left(\bar{\gamma} d^{2} /\left(K_{R}+1\right) \sin ^{2} \theta\right)\right]^{L}} d \theta
\end{aligned}
$$

In this paper, only the error performance expressions of M-PSK in Rician fading channels are presented. The error probabilities in Rayleigh and Nakagami-m fading channels are easily obtained from the above expressions. The Rayleigh fading channel is a special case of Rician fading channel where the Rice factor is equal to zoro, i.e. $K_{R}=0$. In addition, the Nakagami-m fading with the integer parameter $m$ in the $L$-th order diversity channel is equivalent to the Rayleigh channel with the $m L$-th order diversity. Thus, by providing the error performance expressions for Rician fading channels the error performance of the linearly modulated signals in Rayleigh and Nakagami-m fading channels could be easily calculated.

The BER of the DBPSK, DQPSK, and D8PSK in Rician fading channels assuming Gray coding are given as follows [11]:

DBPSK:

$$
B E R_{2 P S K-\text { Rice }}=S E R_{2 P S K-\text { Rice }}=G\left(0, \frac{\pi}{2}, \overline{\gamma_{c}}, L, K_{R}, 1\right)
$$

DQPSK:

$$
B E R_{4 P S K-R i c e}=G\left(0, \frac{\pi}{2}, \overline{\gamma_{b}}, L, K_{R}, 1\right)
$$

where $\overline{\gamma_{b}}=\frac{\overline{\gamma_{c}}}{\log _{2} M}$ in which $M=4$ for DQPSK.

D8PSK:

$$
\begin{aligned}
B E R_{8 P S K-\text { Rice }} & =\frac{1}{3} G\left(0, \frac{7 \pi}{8}, 3 \overline{\gamma_{b}}, L, K_{R}, \sin \frac{\pi}{8}\right) \\
& +\frac{1}{3} G\left(0, \frac{5 \pi}{8}, 3 \overline{\gamma_{b}}, L, K_{R}, \sin \frac{3 \pi}{8}\right)
\end{aligned}
$$

Besides to the models for error performance evaluation of the wireless communication systems over fading channels, few analytical models for BER evaluation in the literature have been developed in which only co-channel interference is considered. A closed-form expression for average BER performance of BPSK was presented in [30] where multiple general identically distributed co-channel interference and 
AWGN exist. In [31] PDF of the co-channel interference due to a QPSK interfering signal and a closedform expression for the bit error probability of QPSK with multiple equally distributed CCI without considering diversity were developed. The authors in [32] presented exact expressions for average BER of DBPSK and DQPSK with GSC diversity in the presence of asynchronous Rayleigh-faded CCI with arbitrary power levels.

The third category of the analytical models of error performance evaluation belongs to the models which assumes both fading desired channels and CCI. In [31] an approximate expressions for average BER of QPSK was presented where the desired channel is either Nakagami-m or Rayleigh faded with Rayleigh CCI. Closed-form expressions for average BER approximation of coherent and differentially coherent BPSK with MRC diversity were derived in [33] where both the desired and interfering channels were assumed to experience Nakagami-m fading. A closed-form expression for exact BER of BPSK was developed in [34] in Rayleigh/Rayleigh desired/interfering channels. The BPSK signal was assumed to be subject of a random number of asynchronous interfering signals with random or unequal average power levels. The authors in [35] derived an exact expressions for average BER of binary coherent and differential PSK in correlated Rician fading channels (having arbitrary and non-identical parameters) with MRC diversity for coherent PSK and EGC diversity for differential PSK. For decreasing the computation complexity the authors also derived approximation expressions in the form of a single integral with finite limits. Closed-form expressions for exact BER of coherent BPSK and coherent QPSK in asynchronous multiple CCI were presented in [36] using a characteristics function method. The desired/interfering channels were considered Nakagami/Nakagami and Nakagami/Rayleigh flat fading for BPSK and QPSK, respectively. The expressions were derived in the form of single integral which is numerically solved.

\section{B. Modeling the error performance by FSMCs}

Apart from the above analytical models for error performance of the wireless communication systems, there is a different modeling approach for the error performance of wireless systems based on the Finite State Markov Chains (FSMCs). The FSMC models have been widely deployed for modeling the error performance of the communication systems in time-varying wireless flat-fading channels. FSMCs are 
appropriate for modeling the communication channels in which the fading channel is correlated and a random process. That is, the channel is a random process that changes over time in a correlated manner. The flat fading channel term indicates that the fading characteristics are independent from the frequency band. Deploying the FSMCs for error performance modeling of wireless systems relies on the notion that the wireless channel condition can be adequately described by a finite number of states. The GilbertElliot Channel (GEC) is known as the basic model of this category, which was developed for modeling the bursty error rate of wireless channels [37], [38]. The proposed models by Gilbert and Elliot were simple two-state Markov chains with memory, along with two Discrete Memoryless Channels (DMCs). The Markov chain in the GEC model is composed of two states of good, G, and bad, B. The channel condition changes from the good state to the bad state with probability of $b$ while $g$ is the probability of moving from the bad state to the good state. The channel input and output are related in the state using two DMCs [39], [37], [38].

The FSMCs can be used for modeling the multichannel wireless communication to support the diversity. As a simple example a wireless system with an antenna diversity of order two in which the channels are independently faded could be modelled using a three-state FSMC; the states represent the cases where both channels are good, the conditions of the channels are different, and both channels are bad [7].

In [40], Vucetic studied the throughput and error performance of adaptive coding algorithms using a three-state FSMC in a time-varying Rician fading channel, in which the states defer by the different Rician factors. The developed FSMC in [41] is considered a major improvement on the employment of the FSMCs for modeling the wireless channels since for the first time the time-varying received SNR of the channel was partitioned into a finite number of intervals so that each interval, hence the average error probability, was associated to a specific state of the FSMC. The chain in this work has been developed for a Rayleigh fading channel, in which the steady state probabilities are calculated based on approximated transition probabilities. In addition, a closed-form expression was derived in the paper for coherent BPSK signals in Rayleigh fading channels.

In the recent years a few FSMCs have been developed in the literature for modeling the error performance of wireless communication systems. The investigations have been deployed in design, analysis 
and performance evaluation of wireless systems. In [42] the authors developed a first-order FSMC to approximate the flat correlated multipath-fading channel to study the effect of Rayleigh fading on the performance of a simple Aloha-based medium access control. The main assumption in a first-order FSMC is that the current channel state is statistically independent of all other past and future channel states. A closed-form expression for packet error rate was given in this work. In [43] the authors develop a closedform expression for error rate of a Rayleigh fading channel using a second order FSMC. The results of their work indicate that deploying higher order FSMC for modeling the wireless channels improves the accuracy of the developed performance-related expressions. The authors in [44] develop a FSMC of an order of higher than one for modeling the wireless channel based on Context Tree Pruning (CTP) algorithm [45]. Closed-form expressions are calculated for packet error rate in Rayleigh and Rician fading channels in presence of AWGN.

It is generally believed in the literature that first order FSMCs are appropriate for slow fading channels while higher order FSMCs are required for accurate modeling of medium-rate and fast fading channels. The error performance of the wireless communication systems was investigated by the first order FSMCs in [46], [47], [48], [49], [50], [51], [52], [53], [54] in Rayleigh fading channels, in [55], [56], [52] in Rician fading channels, and in [56] in Nakagami-m fading channels. In [50] the authors develop closed-from expressions for average BER using first-order FSMC considering BPSK and QPSK. An SNR partitioning mechanism was proposed in [50] to compute the required number and boundaries of the partitions based on the channel conditions. The authors in [56] calculated closed-form expressions for average BER and PER of BPSK and QPSK signals in Rician and Nakagami-m fading channels.

The developed FSMCs in [57], [58] model the wireless channels and compute closed-form expressions for error performance where the diversity techniques are deployed. [57] assumes Rayleigh fading channels and equal gain combining diversity for the wireless communication systems. The FSMC in [58] was developed for a Nakagami fading channel with taking into account SC, MRC, and EGC diversities. There are many studies in the literature which employ the FSMCs for modeling the fading channels of wireless communication systems such as [59], [60], [61], [62], [63], [64], [65], [66], [67], [68], [69], [70]. However, we do not discuss them in this work since they do not either investigate the error performance of the 
wireless communication systems or consider the modulation schemes of the IEEE 802.15.6-based WBANs. Their developed FSMC models can be extended to be applicable for error performance investigation of the WBANs.

The developed first-order FSMC in [64] models time-varying flat fading channels where both amplitude and phase are unknown. They investigate the performance of Rayleigh, Lognormal and Weibull fading channels in terms of steady state probability, state time duration, state transition probability, and level crossing rate. For a more comprehensive discussion about the deployment of FSMCs for modeling the flat fading channels, the interested reader is referred to [39]. In [39], the authors extensively discuss the FSMCs, their deployment history, their applications, comparison of the first-order and higher order FSMCs, amplitude-only and phase only and amplitude-phase FSMC modeling. They also provide a survey of the developed models up to 2007.

In [71], the authors develop a Variable-Length Markov Chain (VLMC) to investigate the performance of a fading channel. A VLMC is a Markovian process whose structure has a sparse memory with some states lumped together. The structure of a VLMC can be represented by a variable-order FSMC with a parsimonious number of transition probabilities [71]. An optimal discretization criterion was developed for the VLMC according to the cost function of fading channel statistics and the transmission policy used in the system. By developing an iterative approach, without proposing a closed-form expression, the error performances of wireless communication systems in Rayleigh and Lognormal fading channels were extensively investigated in their work.

In addition to the above mentioned references, there are a few studies in the literature which deploy the FSMCs in cross-layer performance evaluation or mechanisms for wireless communication systems [72], [73], [65], [56], [74], [68], [75]. The developed models in the studies cannot be directly deployed for WBANs since they assume the mechanisms and characteristics of other types of wireless networks.

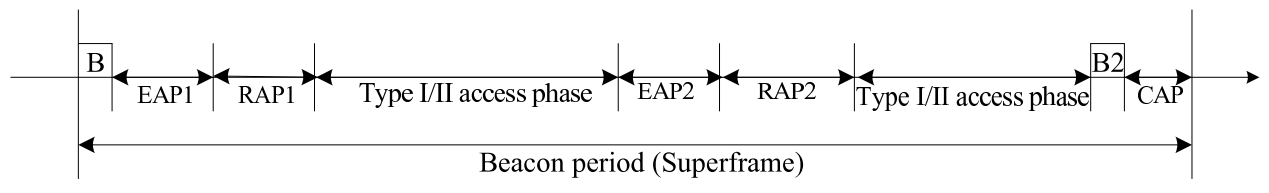

Fig. 1. Layout of access phases in a beacon period (superframe) for beacon mode 
TABLE III

BAN USER PRIORITY MAPPING

\begin{tabular}{|c|c|c|c|c|}
\hline UP & Traffic designation & Frame type & $\mathrm{CW}_{\min }$ & $\mathrm{CW}_{\max }$ \\
\hline 0 & Background (BK) & Data & 16 & 64 \\
\hline 1 & Best effort (BE) & Data & 16 & 32 \\
\hline 2 & Excellent effort (EE) & Data & 8 & 32 \\
\hline 3 & Controlled load (CL) & Data & 8 & 16 \\
\hline 4 & Video (VI) & Data & 4 & 16 \\
\hline 5 & Voice (VO) & Data & 4 & 8 \\
\hline 6 & Media data or network control & Data or Management & 2 & 8 \\
\hline 7 & Emergency or medical event report & Data & 1 & 4 \\
\hline
\end{tabular}

\section{IMPACT OF PHY ERROR MODEL ON MAC PERFORMANCE}

By deploying the WBAN error model, as discussed in the previous sections, impact of channel fading on MAC performance could be investigated. For this purpose BER is calculated based on communication type (modulation/detection), channel fading, co-channel interference, and transmission power, i. e. as a function of Signal to Noise Ratio (SNR) at a receiver. The calculated BER formula is deployed into the MAC performance model to investigate the two layer error performance.

The medium access mechanisms of the IEEE 802.15.6 standard include four categories; random access (connectionless contention-based access), improvised and unscheduled access (connectionless contentionfree access), scheduled access (connection-oriented contention-free access), and medical implant communications service (MICS) band access. The contention-based access methods for obtaining the allocations are either Carrier Sense Multiple Access/Collision Avoidance (CSMA/CA) if narrowband PHY (Physical Layer) is chosen or Slotted Aloha in case of using Ultra-Wideband (UWB) PHY.

For a WBAN which operates based on a contention-based medium access mechanism, such as IEEE 802.15.6 CSMA/CA, the error performance is influenced by two factors, namely frame collisions and BER. If the frame was corrupted by noise or collision the node has to retransmit it if it needs to be acknowledged. The standard allows both acknowledgement and non-acknowledgement modes. However, if the acknowledgement mechanism is not deployed the application reliability decreases. The frame retransmission caused by the collision degrades the WBAN MAC performance, but not uniformly for all nodes. The MAC performance is differently affected by errors based on different user priorities. The user priorities and access phases in CSMA/CA-based WBANs are described as follows:

- According to the IEEE 802.15.6 standard there are 8 different User Priorities (UPs) in a WBAN 
which are differentiated by minimum and maximum Contention Window sizes $\left(\mathrm{CW}_{\min }\right.$ and $\left.\mathrm{CW}_{\max }\right)$, as shown in Table III. The time is divided into beacon periods (superframes) by the hub (the network coordinator). As depicted in Fig. 1 a superframe is divided into Exclusive Access Phases (EAP1 and EAP2), Random Access Phases (RAP1 and RAP2), and Contention Access Phases (CAP), and Type I/II Access Phases. Type I/II APs are indicated for contention-free medium access mechanisms while the other APs are used for contention-based MAC schemes. The EAP periods can be only accessed for transmitting the $\mathrm{UP}_{7}$ frames while all UPs are allowed to access the medium during RAPs or a CAP. The backoff mechanism of the IEEE 802.15.6 standard has been modelled in [76].

A corrupted frame by PHY BER has to be retransmitted, in case of the acknowledgement mechanism deployment. Based on BER and the frame size, we are able to calculate the Packet Error Rate (PER), as $P E R=(1-B E R)^{L}$, in which $L$ represents the frame size. By retransmitting the corrupted frame the non-saturation regions of UPs shrink, as the non-saturation/saturation boundaries move to smaller values. Hence, the WBAN MAC performance is affected by the medium access mechanism and the channel characteristics. Since BER is a function of SNR, increasing the SNR value, by higher transmission power for instance, enhances the WBAN performance. In addition, using larger diversity orders and deployment of appropriate antennas for WBAN nodes the MAC performance could be improved.

The collision probability for different UPs is influenced by a variety of factors including the number of nodes in the network, UPs, CW sizes, data frame arrival rates, the date frame sizes, transmission rates, physical error rate, data frame retransmissions, and even the neighbouring WBANs. Detailed collision probability model can be found in [77], [78]. The standard also allows deployment of RTS/CTS mechanism to access the medium or using the CSMA mechanism without the RTS/CTS mechanism. Our previous studies indicate that deployment of RTS/CTS mechanism also considerably affects the MAC performance. The results in [79] show that RTS/CTS deployment for WBANs is counter-productive and leads to early saturation which is unstable regime. By controlling these factors, the collision probability could be decreased which results in better MAC performance.

In [76] we investigated the MAC performance of IEEE 802.15.6-based WBANs over Rician-faded channels using analytical and simulation models. We assumed Rician-faded channels between the nodes 
and the hub in the network with unequal Rician factors. In our model, the WBAN nodes deploy the QPSK modulation scheme to achieve the highest data frame transmission rate in ISM bands. The obtained BER values for different UPs were used for calculating the data frame error rates of the UPs. We investigated the impacts of channel quality, data frame size, diversity order, data frame arrival rate, access phase lengths, and UPs on error performance of IEEE 802.15.6 CSMA-based WBANs. The results indicate that the diversity order and transmission power parameters can be deployed for achieving the desired WBAN error performance.

For a WBAN which operates based on a contention-free medium access mechanism, the error performance is only affected by BER. Since there is no collision in the network the frames are not corrupted for any other reason than BER. Therefore, by increasing SNR, diversity order and deployment of appropriate antenna, the network MAC performance is improved (but scheduling overhead of corrupted frame increases).

\section{CONCLUSION}

In this work, we discussed channel modeling for IEEE 802.15.6-based WBANs. We also surveyed the research studies which model and formalize the error performance appropriate for developing the WBAN error performance investigation modeling. We also discussed the impact of the PHY error performance on the network MAC performance. The WBAN MAC performance could be improved by two factors of frame collision probability in the MAC layer and BER in the PHY layer. By controlling the effective parameters on these two factors the network MAC performance could be improved. In spite of all performed research in this area, the WBAN error modelling is in early phase and developing new error models for WBANs is a must.

\section{REFERENCES}

[1] Wireless Body Area Networks Standard, IEEE Std. 802.15.6, Feb. 2012.

[2] A. Taparugssanagorn, A. Rabbachin, M. Hmlinen, J. Saloranta, and J. Iinatti, "A review of channel modelling for wireless body area network in wireless medical communications," in Proc. IEEE WPMC, Lapland, Finland, Sep. 2008.

[3] E. N. Farag and M. I. Elmasry, Mixed Signal VLSI Wireless Design Circuits and Systems. Springer US, 1999, ch. Digital Modulation Schemes.

[4] Y. I. Nechayev, P. Hall, I. Khan, and C. C. Constantinou, "Wireless channels and antennas for body-area networks," in Proc. IEEE WONS, Kranjska Gora, Slovenia, Feb. 2010, pp. 137-144.

[5] I. Khan, P. S. Hall, A. A. Serra, A. R. Guraliuc, and P. Nepa, "Diversity performance analysis for on-body communication channels at $2.45 \mathrm{GHz}, "$ IEEE Transactions on Antennas and propagation, vol. 57, pp. 956-963, Apr. 2009.

[6] A. F. Molisch, Wireless Communications. John Wiley and Sons, 2010. 
[7] H. Bai and M. Atiquzzaman, "Error modelling schemes for fading channels in wireless communications: A survey," IEEE Communications Surveys and Tutorials, vol. 5, pp. 2-9, Dec. 2003.

[8] T. Eng, N. Kong, and L. B. Milstein, "Comparison of diversity combining techniques for rayleigh-fading channels," IEEE Transactions on Communications, vol. 44, pp. 1117-1129, Sep. 1996.

[9] M. Z. Win, N. C. Beaulieu, L. A. Shepp, B. F. Logan, and J. H. J. Winters, "On the SNR penalty of MPSK with hybrid selection/maximal ratio combining over i.i.d. rayleigh fading channels," IEEE Transactions on Communications, vol. 51, pp. 1012-1023, Jun. 2003.

[10] M. K. Simon and M. Alouini, "A unified approach to the performance analysis of digital communication over generalized fading channels," Proceedings of the IEEE, vol. 86, pp. 1860-1877, Sep. 1998.

[11] M. G. Shayesteh, "Exact symbol and bit error probabilities of linearly modulated signals with maximum ratio combining diversity in frequency nonselective rician and rayleigh fading channels," IET Communications, vol. 5, pp. 12-26, Jan. 2011.

[12] S. L. Cotton and W. G. Scanlon, "An experimental investigation into the influence of user state and environment on fading characteristics in wireless body area networks at $2.45 \mathrm{GHz}$," IEEE Transactions on Wireless Communications, vol. 8, pp. 6-12, Feb. 2009.

[13] Y. I. Nechayev, P. S. Hall, and Z. H. Hu, "Characterisation of narrowband communication channels on the human body at $2.45 \mathrm{GHz}$," IEEE Microwaves, Antennas \& propagation, vol. 4, pp. 722-732, Jun. 2010.

[14] I. Khan, Y. I. Nechayev, and P. S. Hall, “On-body diversity channel characterization," IEEE Transactions on Antennas and propagation, vol. 58, pp. 573-580, Feb. 2010.

[15] Z. H. Hu, Y. I. Nechayev, P. S. Hall, C. C. Constantinou, and H. Yang, "Measurements and statistical analysis of on-body channel fading at $2.45 \mathrm{GHz}$, , IEEE Antennas and Wireless Propagation Letters, vol. 6, pp. 612-615, Jan. 2007.

[16] Y. I. Nechayev, Z. H. Hu, , and P. S. Hall, "Short-term and long-term fading of on-body transmission channels at $2.45 \mathrm{GHz}$," in Proc. IEEE LAPC, Birmingham, UK, Dec. 2009, pp. 657-660.

[17] S. L. Cotton and W. G. Scanlon, "Measurements and statistical analysis of on-body channel fading at 2.45 GHz," IEEE Antennas Wireless Propagation Letters, vol. 6, pp. 51-55, Mar. 2007.

[18] — " "A statistical analysis of indoor multipath fading for a narrowband wireless body area network," in Proc. IEEE PIMRC, Helsinki, Finland, Sep. 2006, pp. 1-5.

[19] T. T. Tjhung, C. Loo, and N. P. Secord, "BER performance of DQPSK in slow rician fading," IEEE Electronics Letters, vol. 28, pp. 1763-1765, Aug. 1992.

[20] M. Tanda, "Bit error rate of dqpsk signals in slow nakagami fading," IEEE Electronics Letters, vol. 29, pp. 431-432, Mar. 1993.

[21] C. Tellambura and V. K. Bhargava, "Unified error analysis of DQPSK in fading channels," IEEE Electronics Letters, vol. 30, pp. 2110-2111, Dec. 1994.

[22] J. F. Weng and S. H. Leung, "Analysis of DPSK with equal gain combining in nakagami fading channels," IEEE Electronics Letters, vol. 33, pp. 654-656, Apr. 1997.

[23] F. Patenaude, J. H. Lodge, and J. Y. Chouinard, "Error probability expressions for noncoherent diversity in nakagami fading channels," in Proc. IEEE VTC, Phoenix, USA, May 1997, pp. 1484-1487.

[24] M. K. Simon and M. S. Alouini, Digital communications over fading channel. John Wiley, 2005.

[25] D. D. Bevan, V. T. Ermolayev, and A. G. Flaksman, "Coherent multichannel reception of binary modulated signals with dependent rician fading," IEE Proceedings on Communications, vol. 148, pp. 105-111, Apr. 2001.

[26] J. Sun and I. S. Reed, "Linear diversity analysis for M-PSK in rician fading channels," IEEE Transactions on Communications, vol. 51, pp. 1749-1753, 2003.

[27] L. Cao and N. C. Beaulieu, "Closed-form BER results for MRC diversity with channel estimation errors in rician fading channels," IEEE Transactions on Wireless Communications, vol. 4, pp. 1440-1447, 2005.

[28] J. G. Proakis and M. Salehi, Digital Communications (5th Ed.). McGraw-Hill, 2008.

[29] M. G. Shayesteh and A. Aghamohammadi, "On the error probability of linearly modulated signals on the frequency flat rician, rayleigh, and AWGN channels," IEEE Transactions on Communications, vol. 43, pp. 1454-1466, 1995.

[30] M. Chiani, "Analytical distribution of linearly modulated cochannel interferers," IEEE Transactions on Communications, vol. 45, pp. 73-79, Jan. 1997.

[31] _ - "Bandwidth efficient QPSK in cochannel interference and fading," IEEE Transactions on Communications, vol. 43, pp. 24642474, Sep. 1995.

[32] K. A. Hamdi and L. Pap, "Exact BER analysis of binary and quaternary PSK with generalized selection diversity in cochannel interference," IEEE Transactions on Vehicular Technology, vol. 56, pp. 1849-1856, Jul. 2007.

[33] V. A. Aalo and Z. Jingjun, "On the effect of cochannel interference on average error rates in nakagami-fading channels," IEEE Communications Letters, vol. 3, pp. 136-138, May 1999.

[34] K. A. Hamdi, "Exact probability of error of BPSK communication links subjected to asynchronous interference in rayleigh fading environment," IEEE Transactions on Communications, vol. 50, pp. 1577-1579, Oct. 2002.

[35] Y. Ma, T. J. Lim, and S. Pasupathy, "Error probability for coherent and differential PSK over arbitrary rician fading channels with multiple cochannel interferers," IEEE Transactions on Communications, vol. 50, pp. 429-441, Mar. 2002.

[36] K. Sivanesan and N. C. Beaulieu, "Exact BER analyses of nakagami/nakagami CCI BPSK and nakagami/rayleigh CCI QPSK systems in slow fading," IEEE Communications Letters, vol. 8, pp. 45-47, Jan. 2004.

[37] E. N. Gillbert, "Capacity of a bursty-noise channel," Bell System Technology Journal, vol. 39, pp. 1253-1265, Sep. 1960.

[38] E. O. Elliott, "Estimates of error rates for codes on bursty noise channels," Bell System Technology Journal, vol. 42, pp. 1977-1997, Sep. 1963.

[39] P. Sadeghi, R. A. Kennedy, P. B. Rapajic, and R. Shams, "Finite-state markov modelling of fading channels," IEEE Signal Processing Magazine, vol. 25, pp. 57-80, Sep. 2008. 
[40] B. Vucetic, "An adaptive coding scheme for time-varying channels," IEEE Transactions on Communications, vol. 39, pp. 653-663, May 1991.

[41] H. Wang and N. Moayeri, "Finite-state markov channel- a useful model for radio communication channels," IEEE Transactions on Vehicular Technology, vol. 44, pp. 163-171, Feb. 1995.

[42] A. Chockalingam, M. Zorzi, L. Milstein, and P. Venkataram, "Performance of a wireless access protocol on correlated rayleigh-fading channels with capture," IEEE Transactions on Communications, vol. 46, pp. 644-655, May 1998.

[43] M. Chu, D. Goeckel, and W. Stark, "On the design of markov models for fading channal," in Proc. IEEE VTC, Amsterdam, The Netherlands, Sep. 1999, pp. 2372-2376.

[44] F. Babich, O. Kelly, and G. Lombardi, "Generalized markov modelling for flat fading," IEEE Transactions on Communications, vol. 48, pp. 547-551, Apr. 2000.

[45] _ _ "A context-tree based model for quantized fading," IEEE Communications Letters, vol. 3, pp. 46-48, Feb. 1999.

[46] C. Tan and N. Beaulieu, "On first-order markov modelling for the rayleigh fading channel," IEEE Transactions on Communications, vol. 48, pp. 2032-2040, Dec. 2000.

[47] A. Karmokar, D. Djonin, and V. Bhargava, "Optimal and suboptimal packet scheduling over correlated time varying flat fading channels," IEEE Transactions on Wireless Communications, vol. 5, pp. 446-456, Feb. 2006.

[48] _ - "POMDP-based coding rate adaptation for type-i hybrid ARQ systems over fading channels with memory," IEEE Transactions on Wireless Communications, vol. 5, pp. 3512-3523, Dec. 2006.

[49] P. Sadeghi and P. Rapajic, "Capacity analysis for finite-state markov mapping of flat-fading channels," IEEE Transactions on Communications, vol. 53, pp. 833-840, May 2005.

[50] D. Dalalah, L. Cheng, and G. Tonkay, "Modeling end-to-end wireless lossy channels: A finite-state markov approach," IEEE Transactions on Wireless Communications, vol. 7, pp. 1236-1243, Apr. 2008.

[51] M. K. Chang, S. Y. Lee, C. H. Chien, and C. H. Kuo, "Performance analysis and modelling of single-step power control in finite state markov channel under different feedback channels," IEEE Transactions on Communications, vol. 58, pp. 1280-1290, Apr. 2010.

[52] Q. Pan, S. Liu, M. Xu, and C. Jia, "Finite-state markov model for the aeronautical channel," in Proc. IEEE WiCom, Beijing, China, Sep. 2009, pp. 1-4.

[53] J. M. Park and G. U. Hwang, "Mathematical modelling of rayleigh fading channels based on finite state markov chains," IEEE Communications Letters, vol. 13, pp. 764-766, 2009.

[54] P. Sadeghi and P. Rapajic, "On information rates of time-varying fading channels modelled as finite-state markov channels," IEEE Transactions on Communications, vol. 56, pp. 1268-1278, Aug. 2008.

[55] F. Babich and G. Lombardi, "A markov model for the mobile propagation channel," IEEE Transactions on Vehicular Technology, vol. 49, pp. 63-73, Jan. 2000.

[56] S. Vassilaras, "A cross-layer optimized adaptive modulation and coding scheme for transmission of streaming media over wireless links," Wireless Network Journal, vol. 16, pp. 903-914, May 2010.

[57] Y. Guan and L. Turner, "Generalised FSMC model for radio channels with correlated fading," IEEE Proceedings on Communications, vol. 146, pp. 133-137, Apr. 1999.

[58] C. Iskander and P. Mathiopoulos, "Fast simulation of diversity nakagami fading channels using finite-state markov models," IEEE Proceedings on Broadcasting, vol. 49, pp. 269-277, Sep. 2003.

[59] A. Hoang and M. Motani, "Buffer and channel adaptive transmission over fading channels with imperfect channel state information," in Proc. IEEE WCNC, Atlanta, USA, Mar. 2004, pp. 1891-1896.

[60] J. Xiao, J. Qiu, and S. Cheng, "A joint adaptive packet size and modulation scheme combined with SR-ARQ over correlated fading channels," in Proc. IEEE WiCOM, Wuhan, China, Sep. 2005, pp. 478-483.

[61] L. Johnston and V. Krishnamurthy, "Opportunistic file transfer over a fading channel: A POMDP search theory formulation with optimal threshold policies," IEEE Transactions on Wireless Communications, vol. 5, pp. 394-405, Feb. 2006.

[62] H. Wang and N. B. Mandayam, "Opportunistic file transfer over a fading channel under energy and delay constraints," IEEE Transactions on Communications, vol. 53, pp. 632-644, Apr. 2005.

[63] C. Pimentel, T. H. Falk, and L. Lisboa, "Finite-state markov modelling of correlated rician fading channels," IEEE Transactions on Vehicular Technology, vol. 53, pp. 1491-1501, Sep. 2004.

[64] V. Nithya and V. Bhaskar, "Finite-state markov channel modelling under jointly varying amplitude and phase in time-varying flat fading channels," IET Communications, vol. 5, pp. 1237-1245, Jun. 2011.

[65] Y. Wei, F. R. Yu, and M. Song, "Distributed optimal relay selection in wireless cooperative networks with finite-state markov channels," IEEE Transactions on Vehicular Technology, vol. 59, pp. 2149-2158, Jun. 2010.

[66] G. Verticale, "A closed-form expression for queuing delay in rayleigh fading channels using stochastic network calculus," in Proc. ACM Q2SWinet, Tenerife, Spain., Oct. 2009, pp. 8-12.

[67] T. Li and O. M. Collins, "A successive decoding strategy for channels with memory," IEEE Transactions on Information Theory, vol. 53, pp. 628-646, Feb. 2007.

[68] R. Zhang and L. Cai, "Joint AMC and packet fragmentation for error control over fading channels," IEEE Transactions on Vehicular Technology, vol. 59, pp. 3070-3080, Jul. 2010.

[69] H. Al-Lawati, F. Alajaji, and C. Pimentel, "On symbol versus bit interleaving for block-coded binary markov channels," IEEE Transactions on Vehicular Technology, vol. 59, pp. 2582-2588, Jun. 2010.

[70] L. Zhong, F. Alajaji, and G. Takahara, "A model for correlated rician fading channels based on a finite queue," IEEE Transactions on Vehicular Technology, vol. 57, pp. 79-89, Jan. 2008.

[71] W. Kumwilaisak, C. C. J. Kuo, and D. Wu, "Fading channel modelling via variable-length markov chain technique," IEEE Transactions on Vehicular Technology, vol. 57, pp. 1338-1358, May 2008. 
[72] — - "Joint rate and power adaptation for type-i hybrid ARQ systems over correlated fading channels under different buffer-cost constraints," IEEE Transactions on Vehicular Technology, vol. 57, pp. 421-435, Jan. 2008.

[73] A. T. Hoang and M. Motani, "Cross-layer adaptive transmission: Optimal strategies in fading channels," IEEE Transactions on Communications, vol. 56, pp. 799-807, May 2008.

[74] J. Xiao, S. Zou, J. Qiu, and S. Cheng, "A cross-layer adaptive transmission scheme combined with SR-ARQ over correlated fading channels," Journal of Computers and Electrical Engineering, vol. 34, pp. 324-337, Jul. 2008.

[75] A. K. Karmokar and V. K. Bhargava, "Performance of cross-layer optimal adaptive transmission techniques over diversity nakagami-m fading channels," IEEE Transactions on Communications, vol. 57, pp. 3640-3652, Dec. 2009.

[76] S. Rashwand, J. Misic, and V. B. Misic, "MAC performance modeling of IEEE 802.15.6-based WBANs over rician-faded channels," in Proc. the IEEE International Conference on Communications (ICC'12), Ottawa, Canada, Jun. 2012, pp. $5462-5467$.

[77] S. Rashwand and J. Misic, "Effects of access phases lengths on performance of IEEE 802.15.6 CSMA/CA mechanism," Journal of Computer Networks (COMNET), vol. 56, pp. 2832-2846, Aug. 2012.

[78] — "Performance evaluation of IEEE 802.15.6 under non-saturation condition," in Proc. the IEEE Global Telecommunications Conference (Globecom11), Houston, Texas, US., Dec. 2011, pp. 1-6.

[79] S. Rashwand, J. Misic, and V. Misic, “Analysis of CSMA mechanism of IEEE 802.15.6," IEEE Transactions on Parallel and Distributed Systems, 2013, Under Revision. 